\title{
La competitividad externa de Canadá y de sus socios del TLCAN revelada en los índices internacionales
}

$\mathrm{D}$ esde las últimas décadas del siglo $\mathrm{xx}$, los procesos vinculados a la globalización económica, aunados a las reformas estructurales emprendidas en el ámbito nacional, han incrementado la competencia entre economías nacionales. El nuevo modelo económico, implementado de manera casi universal, ha reducido el papel tradicional del Estado por medio de la desreglamentación y de la privatización de empresas públicas.

En el ámbito de las relaciones económicas externas destacan dos fenómenos: por un lado, la apertura del comercio exterior y la importancia creciente otorgada a la demanda externa en tanto motor del desarrollo han fomentado la búsqueda de un mayor desempeño exportador; por otro, las políticas económicas han revalorizado el papel de la inversión extranjera directa no sólo como una fuente de recursos financieros sino como un medio de acceso a tecnologías más avanzadas, que permitan elevar la productividad en sectores claves, o como una llave de entrada a mercados externos.

Este nuevo enfoque de las políticas nacionales se ha combinado con las estrategias productivas de las grandes empresas, fortaleciendo las redes productivas globalizadas y la penetración de éstas en los mercados internos por medio de la adquisición de empresas locales y, con frecuencia, empresas públicas. Estos procesos han tenido eco a nivel teórico, pues el análisis de las nuevas corrientes de intercambio comercial, en particular en el ámbito manufacturero, ha suscitado el surgimiento de nuevas teorías de comercio exterior que han identificado, al lado de las tradiciona-

* Investigadora del Departamento de Estudios del Pacífico, de la Universidad de Guadalajara. les ventajas comparativas, las ventajas competitivas, que pueden ser creadas y nutridas y cuya evolución explica la trayectoria internacional de las distintas economías en la producción de determinados bienes y servicios comerciables.

En consecuencia, se han ido creando una serie de indicadores e índices internacionales que tratan de dar cuenta empíricamente del grado de competitividad internacional de las economías nacionales. Algunos de estos instrumentos han sido elaborados por organismos multilaterales, como es el caso de la base de datos de 49 indicadores de competitividad recolectados por el Banco Mundial, por medio de su departamento de desarrollo del sector privado, o de los índices calculados por la UNCTAD para dar cuenta del desempeño y potencial de las economías nacionales en tanto receptoras de inversión extranjera directa (IED).

Otros más fueron concebidos por consultoras o fundaciones privadas, publicándose en forma anual informes que recogen los valores alcanzados por las distintas economías incluidas, a las que clasifican en función de su mayor o menor competitividad internacional; entre los más relevantes, cabe citar: el Anuario de la competitividad mundial (World Competitiveness Yearbook, wcy) publicado por la escuela de negocios suiza IMD; el Informe sobre la competitividad global (Global Competitiveness Report, GCR), publicado por el Foro Económico Mundial (World Economic Forum, wEF), y, con un objetivo más acotado, el Índice de libertad económica (Index of Economic Freedom) del "think-tank" conservador estadounidense Heritage Foundation. ${ }^{1}$

Este texto se propone analizar los resultados arrojados por una selección de estos ín- 
dices e indicadores en el caso de Canadá, contrastándolos con la posición de sus socios del Tratado de Libre Comercio de América del Norte (TLCAN). Para ello, se presentarán brevemente los objetivos y las principales características de estos instrumentos, abordando luego su aplicación al caso de Canadá, Estados Unidos y México.

\section{Presentación de los índices de competitividad internacional}

Esta presentación se abocará a un breve análisis de cuatro series de índices para los cuales se dispone de información más reciente: los índices del wCY, del GCR, de libertad económica y de la UNCTAD. Sus propósitos difieren: los dos primeros son los más amplios en cuanto al número de variables incluidas y se proponen un objetivo común: estimar la competitividad global de las economías revisadas. El tercero está más enfocado a medir el grado de libertad económica, que sólo constituye un elemento de la competitividad, mientras que los índices calculados por la UNCTAD tienen el propósito específico de medir el papel actual y el potencial de atracción de la IED en las economías nacionales.

Definición y medición de la competitividad externa en los índices del WCY y del GCR

Aun cuando sus objetivos y criterios muy generales coinciden, el wCY y el GCR difieren tanto en la metodología aplicada como en el rango de países incluidos en la muestra. El objetivo declarado del wcy consiste en "analizar y clasificar la habilidad de las naciones para crear y mantener un ambiente que sustente la competitividad de sus empresas" (RosseletMcCaulay), lo cual subraya la diferencia entre las nociones de competitividad de las empresas y competitividad de las naciones, asumiéndose que "la creación de valor sólo se da en el seno de una empresa" y que "el ambiente de una nación puede dificultar o sustentar este proceso a través de sus políticas" (Garelli, 2003: 702). Los autores distinguen el concepto de competitividad nacional del de desempeño económico, atribuyendo al primero una "dimensión de sustentabilidad de largo plazo" (ibid.). ${ }^{2}$ Como tal, es un concepto dinámico, que no sólo toma en cuenta las variables tradicionalmente consideradas (crecimiento, exportaciones, infraestructura física) sino otras como la educación, el conocimiento, los bienes intangibles, la infraestructura tecnológica y los sistemas de valores de una nación. El contexto de los hechos, como las dotaciones de recursos o los eventos naturales sobre los cuales el ser humano tiene un grado de control mínimo, y de las políticas, que pueden ser alteradas, definen entonces el margen de maniobra del cual dispone un país para establecer el marco competitivo adecuado.

Para evaluar la competitividad de las naciones seleccionadas, el anuario maneja un total de 320 criterios: de éstos, 127 son datos estadísticos utilizados para el cálculo del índice agregado, a partir del cual constituyen alrededor de las dos terceras partes de la ponderación, 116 son variables cualitativas recogidas por medio de una encuesta anual de opinión aplicada entre ejecutivos (una tercera parte de la ponderación del índice) y 78 son datos estadísticos presentados como parte del contexto general del anuario. Los criterios son reagrupados en 20 categorías distintas, las cuales 
conforman a su vez cuatro grandes clases de factores de competitividad, de los que se asume que definen generalmente la calidad del contexto nacional (ver cuadro 1). Cada una de las categorías así conformadas reciben una misma ponderación ( $5 \%$ del índice), de lo cual se deriva que los cuatro factores generales de competitividad tienen cada uno un peso idéntico de $25 \%$ del índice agregado. ${ }^{3}$

En el anuario se incluyen un total de 59 economías, divididas en dos grupos que toman en cuenta el factortamaño: por un lado las economías de más de 20 millones de habitantes y por otro aquéllas con poblaciones menores a los 20 millones. En efecto, los autores del anuario consideran que "las grandes economías muy pobladas presentan un modelo competitivo diferente del de las más pequeñas" (RosseletMcCaulay). Un rasgo específico adicional del wCy es que incluye tanto economías nacionales como algunas de las economías regionales subnacionales más relevantes en el ámbito mundial, las europeas en particular.

En contraste con el wcy, el Informe sobre la competitividad global, elaborado por el Foro Económico Mundial abarca una mayor cantidad de economías: 80 en 2002 y 75 en 2001, ubicadas en un conjunto único, con algunas consideraciones específicas para las economías en desarrollo. El procedimiento aplicado para estimar la competitividad parte de un enfoque compartido con el WCY; se estima que "la competitividad mantiene una relación directa con el nivel de vida de una nación" (Porter: 23), y se destacan dos grandes componentes, evaluados a través de dos índices distintos. Por un lado, las condiciones macroeconómicas, políticas, sociales y legales se hallan recogidas en un índice de competitividad del crecimiento. Éstas constituyen elementos necesarios mas no suficientes para una economía exitosa, ya que "proveen la oportunidad de crear riqueza pero no la crean por sí mismas" (ibid.). Los criterios que sí indican la capacidad de creación de riqueza están incluidos en el índice de competitividad microeconómica, pues se considera que "la riqueza de hecho es creada en el nivel microeconómico de una economía, y se enraíza en la sofisticación de las estrategias y de las prácticas operativas de las empresas, así como en la calidad del contexto microeconómico de negocios en el cual las empresas de una nación compiten" (ibid.). $\mathrm{Al}$ igual que en el caso anterior, ambos índices combinan datos estadísticos con información cualitativa recogida en el marco de encuestas anuales de opinión de ejecutivos.

El índice de competitividad del crecimiento examina las fuentes del crecimiento económico, combinando tres tipos de factores considerados esenciales para un crecimiento sustentable de largo plazo: la tecnología, las instituciones públicas y el contexto macroeconómico, los cuales dan lugar a tres subíndices. La ponderación que reciben estos factores difiere en función del lugar que ocupan las economías nacionales en el entorno mundial. Para las economías desarrolladas consideradas en el núcleo de los innovadores centrales ${ }^{4}$ la ponderación del factor tecnológico es mayor: este subíndice explica la mitad del índice agregado contra una cuarta parte para cada uno de los otros factores. En el caso de las demás economías, cada subíndice recibe una ponderación idéntica, explicando cada

\section{Cuadro 1}

Componentes del índice de competitividad del anuario de competitividad mundial

\begin{tabular}{llll}
$\begin{array}{c}\text { Desempeño económico } \\
\text { (75 criterios) }\end{array}$ & $\begin{array}{c}\text { Eficiencia gubernamental } \\
(81 \text { criterios })\end{array}$ & $\begin{array}{c}\text { Eficiencia de los negocios } \\
(69 \text { criterios })\end{array}$ & $\begin{array}{c}\text { Infraestructura } \\
(96 \text { criterios })\end{array}$ \\
\hline Economía interna & Finanzas públicas & Productividad & Infrestructura básica \\
Comercio internacional & Política fiscal & Mercado de trabajo & $\begin{array}{l}\text { Infraestructura tecnológica } \\
\text { Infraestructura científica }\end{array}$ \\
Emversión internacional & Marco institucional & Financiamiento & Salud y medio ambiente \\
Legislación de negocios & Prácticas de gestión & Actitudes y valores & Educación
\end{tabular}

Fuente: Suzanne Rosselet-McCaulay. "Methodology and Principles of Analysis", en http://www01.imd.ch/wcy, tablas 1 y 2. 
uno una tercera parte del índice agregado (Cornelius et al.: 20). ${ }^{5}$

El índice de competitividad microeconómica, calculado desde 1998, destaca la importancia de estos factores para un crecimiento sustentable, recalcada por las pruebas estadísticas y econométricas realizadas por los autores, las cuales muestran que éstos explicarían el $81 \%$ de las variaciones en el nivel del PIB per cápita de los países. La importancia de estos factores radica en que determinan la productividad nacional, fuente a su vez de la competitividad y la prosperidad (Porter: 25). El índice se calcula como promedio ponderado de dos subíndices que señalan dos aspectos estrechamente relacionados entre sí: el grado de sofisticación de la estrategia y de las operaciones de las firmas (con una ponderación de $37 \%$ del valor del índice), y la calidad del entorno microeconómico de los negocios (63\% de la ponderación total). ${ }^{6}$

En suma, las estimaciones de competitividad elaboradas por el IMC y por el WEF difieren tanto en la metodología empleada como en la muestra de economías incluidas. La ponderación del índice del wcy y de los dos índices del GCR indican que el primero otorga un peso relativamente más grande a las condiciones generales que propician el crecimiento económico y la competitividad externa, mientras que la tecnología y el entorno microeconómico reciben una ponderación mayor en el caso del GCR.

\section{Los índices de libertad económica (Heritage Foundation) y de atracción de IED (UNCTAD)}

Estos índices persiguen objetivos disímiles y más parciales que los presentados en la sección anterior. El primero busca analizar el grado de libertad con el cual se emprenden las actividades de negocios y clasificar las economías nacionales en función de este criterio; en otros términos, se centra en un componente específico de la competitividad internacional: las políticas de gobierno, cuyo carácter liberal es considerado por los autores como necesario para el crecimiento económico. El concepto de libertad económica utilizado es exigente, pues se define como "la ausencia de coerción o restricción pública sobre la producción, la distribución o el consumo de bienes y servicios más allá de la necesaria a los ciudadanos, para proteger y conservar la libertad en sí" (Heritage Foundation, 2003: cap. 5). Para ello se estudian cincuenta variables económicas, reunidas a su vez en diez factores, que reciben una ponderación idéntica: política comercial, carga fiscal del gobierno, intervención pública en la economía, política monetaria, flujos de capitales e inversión extranjera, finanzas y banca, precios y salarios, derechos de propiedad, regulación y actividad del mercado negro (ibid.). Cada criterio recibe un puntaje comprendido entre 1 (menor intervención del gobierno o mayor libertad económica) y 5 (mayor intervención pública y menor libertad), los cuales permiten calcular el índice agregado, un método que ciertamente parece conceder más campo a la apreciación subjetiva de los autores que en los casos anteriores. ${ }^{7} \mathrm{El}$ índice abarca 161 economías y la comparación año tras año del valor del índice para una economía determinada permite estimar el avance de las reformas liberalizadoras estimado por los autores del índice.

El objetivo de tres índices elaborados por la UNCTAD es también acotado: éstos buscan ilustrar el grado de atracción, realizado y potencial, de una economía para la IED. El primero y el más antiguo es el índice de transnacionalización de una economía, que procura dar cuenta del papel desempeñado por la IED en la economía nacional. El índice se calcula como un promedio simple de cuatro indicadores de la importancia relativa de la IED: 1) las entradas (flujos) de IED como porcentaje de la formación bruta de capital fijo promediadas sobre un período de tres años; 2) el acervo (stock) de IED en el país como porcentaje del PIB; 3) el valor agregado de las empresas extranjeras como porcentaje del PIB, y 4) el empleo de las empresas extranjeras como porcentaje del empleo total (UNCTAD, 2000). 


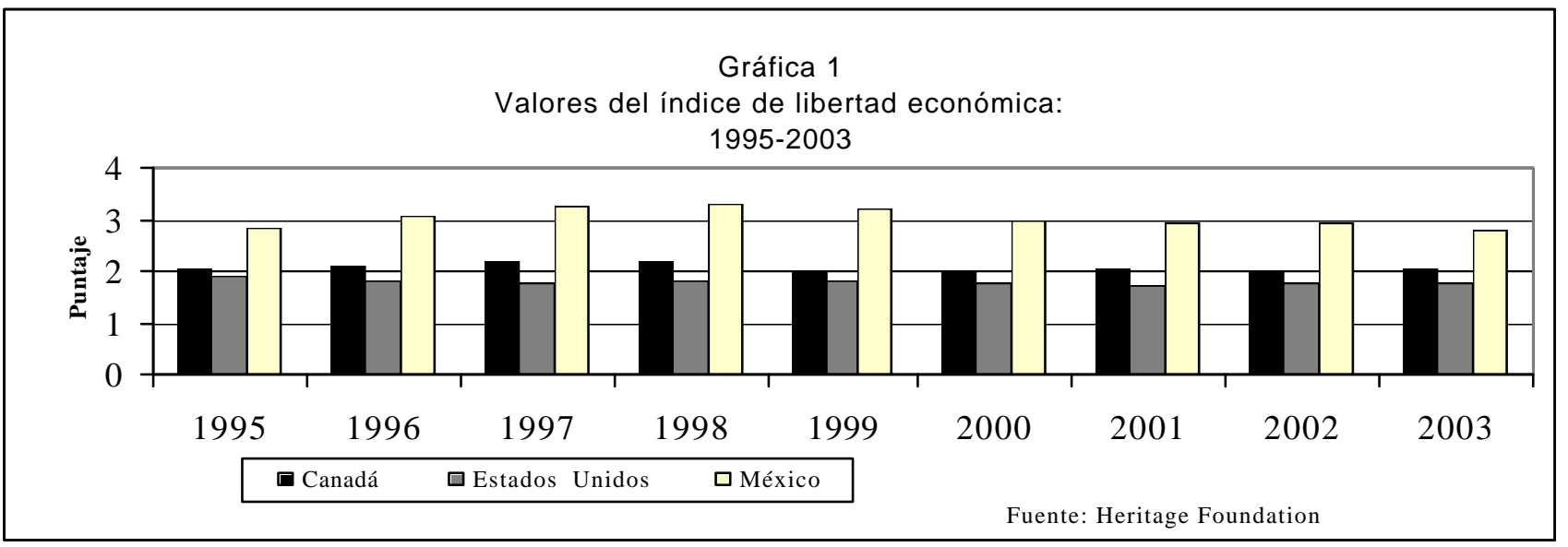

Dos índices más recientes resultan aún más atractivos para los propósitos de este artículo, pues permiten comparar el desempeño actual y potencial de la IED en una economía nacional, estrechamente relacionados con el grado de competitividad del país. En primer lugar, el índice de desempeño de la IED en el país, introducido en el informe 2001, procura medir si una economía recibe flujos de IED proporcionales a su tamaño relativo, a través del cálculo de la razón flujos recibidos por el país/ flujos mundiales dividida por la razón PIB del país/PIB mundial, señalando para valores superiores (inferiores) a 1, que el país recibe proporcionalmente mayores (menores) flujos de IED que su participación al PIB mundial permitiera predecir.
En segundo lugar, el índice de potencial de la IED en el país busca estimar el potencial nacional de atracción de IED. Este índice se obtiene al promediar los valores adquiridos por ocho indicadores susceptibles de afectar la atracción de IED; éstos son en primer lugar tres indicadores macroeconómicos: el PIB per cápita, que subraya el tamaño y el grado de sofisticación de la demanda interna; el crecimiento del PIB real promedio de los diez últimos años, que predice el tamaño futuro de la economía nacional, y la proporción de las exportaciones en relación con el PIB, como proxy del grado de apertura de la economía. El índice incluye luego cuatro indicadores de infraestructura física y humana: la densidad de líneas telefónicas y el uso per cápita de ener-

Cuadro 2

Índice de libertad económica

\begin{tabular}{lccc}
\hline & Canadá & $\begin{array}{c}\text { Estados } \\
\text { Unidos }\end{array}$ & México \\
\hline Política comercial & 2 & 2 & 2 \\
Carga fiscal & 4 & 3.5 & 3.5 \\
Intervención del gobierno & 2.5 & 2 & 3 \\
Política monetaria & 1 & 1 & 3 \\
Inversión extranjera & 3 & 2 & 3 \\
Banca y finanzas & 2 & 1 & 2 \\
Precios y salarios & 2 & 2 & 2 \\
Derechos de propiedad & 1 & 1 & 3 \\
Regulación & 2 & 2 & 3 \\
Mercado negro & 1 & 1.5 & 3.5 \\
& & & \\
Posición & 18 & 6 & 56 \\
Puntaje & 2.05 & 1.8 & 2.8 \\
Categoría & Libre & Libre & Predominan- \\
& & & temente libre \\
\hline
\end{tabular}

Fuente: Heritage Foundation, Economic Freedom Index 2003. 
gía comercial, que estiman la calidad de la infraestructura requerida por los inversionistas; los gastos de investigación y desarrollo como porcentaje del ingreso nacional bruto, que señala las capacidades tecnológicas y de innovación, y la participación de los estudiantes del nivel terciario en la población total, como proxy del capital humano. Finalmente, se incluye también un indicador del riesgo-país, es decir de los riesgos políticos y comerciales asociados a cada economía (UNCTAD, 2002: 34-36). La comparación de estos dos últimos índices, calculados para 83 economías, ${ }^{8}$ permite entonces estimar el desempeño relativo actual y futuro de cada economía en tanto receptora de IED, así como su evolución en el tiempo.

\section{Un análisis de la competitividad internacional de Canadá en relación con Estados Unidos y México}

Esta sección sigue en sus grandes líneas el plan del apartado anterior, es decir, que se hará en primer lugar un análisis de la competitividad externa de Canadá, para luego estudiar de manera más específica la posición de Canadá en tanto receptor de IED. En la comparación de las respectivas posiciones competitivas de las tres economías del TLCAN, cabrá matizar los resultados tomando en cuenta sus diferentes niveles de desarrollo, específicamente en el caso de México, cuyo estadio de desarrollo difiere de sus socios y, como es obvio, implica otros desafíos.

Cabe iniciar el análisis señalando que en la actualidad las tres economías de América del Norte comparten fundamentalmente un enfoque liberal de la política económica. Esto queda señalado por el índice de libertad eco- nómica, que ubica a Canadá y Estados Unidos en el grupo de países libres, mientras México se considera fundamentalmente libre, al haber elevado el grado de libertad de sus políticas en los últimos años, después de alcanzar un "pico" de intervención en 1998-1999 (gráfica 1). La cuadro 2 permite observar que si Estados Unidos es considerado la economía más libre de las tres, Canadá presenta grados de libertad elevados en los ámbitos de la política monetaria y los derechos de propiedad, así como un nivel muy bajo de mercado negro, un hecho constatado también en los índices que veremos a continuación. Sus puntos más débiles, dentro de este enfoque, radicarían en las áreas de carga fiscal, inversión extranjera e intervención del gobierno, donde Estados Unidos presenta mejores récords. En general, México presenta niveles de libertad menores en todos los ámbitos, siendo la política comercial, la banca y los precios y salarios los más liberalizados.

Ahora bien, ¿qué resultado arrojan las estimaciones más amplias de competitividad internacional de las tres economías? El wcy ubica a Canadá en la segunda posición competitiva entre treinta economías de más de 20 millones de habitantes en el período 1999-2002, justo después de Estados Unidos, deslizándose al tercer puesto en 2003, después de Australia. En términos generales, este índice señala por lo tanto que Canadá y Estados Unidos han mantenido elevados niveles de competitividad; en contraste México habría perdido competitividad, al descender de la posición 14 a la 24 (cuadro 3). La desagregación del índice en sus cuatro componentes principales para el año 2003 permite precisar las respectivas fortalezas y debilidades de la posición competitiva de cada economía (cuadro

Cuadro 3

Informe sobre la competitividad mundial 2003: rangos agregados

$1999-2003$

\begin{tabular}{lccccc}
\hline & 1999 & 2000 & 2001 & 2002 & 2003 \\
\hline Canadá* & 2 & 2 & 2 & 2 & 3 \\
Estados Unidos* & 1 & 1 & 1 & 1 & 1 \\
México* $^{*}$ & 14 & 14 & 15 & 19 & 24 \\
\hline
\end{tabular}

* En un grupo de 30 economías con una población que supera los 20 millones de habitantes.

Fuente: IMD: World Competitiveness Yearbook 2003. 
Cuadro 4

Rangos del informe sobre la competitividad mundial, 2002-2003

\begin{tabular}{|c|c|c|c|c|c|c|c|c|c|c|c|c|c|c|c|}
\hline & \multicolumn{3}{|c|}{ Rango agregado } & \multicolumn{3}{|c|}{ Desempeño económico } & \multicolumn{3}{|c|}{ Eficiencia del gobierno } & \multicolumn{3}{|c|}{ Eficiencia en los negocios } & \multicolumn{3}{|c|}{ Infraestructura } \\
\hline & 20 & & 2002 & 20 & & 2002 & & & 2002 & 200 & & 2002 & 200 & & 2002 \\
\hline & Puntaje & Rango & & Puntaje & Rango & & Puntaje & Rango & & Puntaje & Rango & & Puntaje & Rango & \\
\hline Canadá* & 84.1 & 3.0 & 2.0 & 68.4 & 6.0 & 5.0 & 76.8 & 4.0 & 3.0 & 82.2 & 3.0 & 2.0 & 82.0 & 2.0 & 3.0 \\
\hline Estados Unidos* & 100.0 & 1.0 & 1.0 & 99.8 & 1.0 & 1.0 & 78.2 & 2.0 & 1.0 & 92.7 & 1.0 & 1.0 & 100.0 & 1.0 & 1.0 \\
\hline México* & 33.3 & 24.0 & 19.0 & 42.2 & 17.0 & 18.0 & 43.7 & 16.0 & 13.0 & 26.1 & 25.0 & 20.0 & 21.3 & 29.0 & 23.0 \\
\hline
\end{tabular}

4). Así, para el wcy, Canadá tiene como puntos más fuertes su infraestructura y el grado de eficiencia en los negocios, mientras el desempeño económico constituye un punto débil. Por su parte, Estados Unidos predomina en estos tres criterios, siendo relativamente la eficiencia gubernamental su único "punto flaco", pues se ubica en segunda posición después de Australia. En cuanto a México, sus debilidades se ubican en las áreas de infraestructura y eficiencia de los negocios, observándose una mejoría entre 2002 y 2003 en una sola área: el desempeño económico.

El panorama mostrado por los dos índices del weF difiere del que se acaba de comentar. La evolución de las posiciones competitivas de las tres economías entre 2001 y 2002 en el índice de competitividad del crecimiento muestra un retroceso neto de Canadá, desde la tercera hasta la octava posición, y uno más moderado de México (del puesto 42 al 45) mientras Estados Unidos recupera su primer lugar. El cuadro 5 nos muestra que este deslizamiento de Canadá se debe sobre todo a una pérdida de competitividad relativa en el área tecnológica, la cual, como hemos visto, tiene una elevada ponderación en este índice (50\% para la categoría de economías innovadoras centrales). Es su primacía en esta área la que ubica a Estados Unidos en el primer nivel mundial, a pesar del deterioro de su ya bajo desempeño en el índice parcial de instituciones públicas (del rango 12 al 16). Los valores de los subíndices para México evidencian tendencias contradictorias: una mejoría en el área del contexto macroeconómico, que se opone a una degradación en el área tecnológica.

La desagregación de los componentes de estos subíndices permite algunas constataciones interesantes (cuadro 6). Los valores señalan que si Canadá tiene un desempeño macroeconómico intermedio con respecto a sus socios del TLCAN, goza sin embargo de un mayor grado de estabilidad macroeconó-mica (rango 13, contra el 46 de Estados Unidos y el 60 de México), presentando su mayor debilidad en el gasto de gobierno (rango 52, contra el 12 de Estados Unidos y el 4 de México). La calidad de las instituciones públicas, el primer punto fuerte de Canadá en este índice, se atribuye a la calidad del marco legal, pero sobre todo al bajo nivel de corrupción, un área donde decididamente Canadá tendría enseñanzas que compartir con sus dos socios, clasificados en los lugares 20 (Estados Unidos) y 47 (México). Por último, en el área tecnológica, donde sobresale Estados Unidos, Canadá ocupa la octava posición entre las 74 economías revisadas, siendo precedido de Taiwan, Finlandia, Suecia, Japón, Israel y Suiza. México, cuya situación en esta área no es directamente com-

Cuadro 5

Indicadores de competitividad del crecimiento, 2002-2003

\begin{tabular}{|c|c|c|c|c|c|c|c|c|}
\hline \multirow[t]{3}{*}{ País } & \multirow{2}{*}{\multicolumn{2}{|c|}{$\begin{array}{c}\text { Clasificación } \\
\text { de la competitividad } \\
\text { del crecimiento }\end{array}$}} & \multicolumn{6}{|c|}{ Índices parciales } \\
\hline & & & \multicolumn{2}{|c|}{$\begin{array}{c}\text { Clasificación del } \\
\text { contexto macroeconómico }\end{array}$} & \multicolumn{2}{|c|}{$\begin{array}{l}\text { Clasificación de las } \\
\text { instituciones públicas }\end{array}$} & \multicolumn{2}{|c|}{$\begin{array}{c}\text { Rango en el } \\
\text { índice tecnológico }\end{array}$} \\
\hline & 2002 & 2001 & 2002 & 2001 & 2002 & 2001 & 2002 & 2001 \\
\hline Canadá & 8 & 3 & 12 & 13 & 9 & 11 & 8 & 2 \\
\hline Estados Unidos & 1 & 2 & 2 & 7 & 16 & 12 & 1 & 1 \\
\hline México & 45 & 42 & 21 & 36 & 58 & 55 & 47 & 36 \\
\hline
\end{tabular}

NB: 74 países incluidos en 2002 y 75 en 2001; la metodología aplicada ha experimentado cambios entre 2001 y 2002.

Fuente: World Economic Forum, Global Competitiveness Report 2002. 
parable, debido a que no forma parte del reducido grupo de economías innovadoras centrales, muestra debilidades en cuanto a la innovación, compensadas por una mejor posición en la transferencia de tecnologías.

Un punto fuerte de la economía canadiense, que no figura en los cuadros presentados aquí, se refiere a la sustentabilidad medioambiental, donde ocupa un destacado tercer lugar entre 74 economías, mientras Estados Unidos se ubica en la undécima posición y México en el puesto 73, lo que recalca uno de los mayores problemas que el país tendrá que resolver en un futuro no muy lejano. ${ }^{9}$

En el nivel microeconómico (cuadro 7), los resultados hallados por el wCR subrayan la primacía de Estados Unidos frente a sus dos socios. Entre 1998 y 2002, Estados Unidos ha ocupado de manera continua el primer o el segundo puesto tanto en el índice total como en los subíndices que lo componen, mientras que Canadá y México habrían perdido competitividad. En efecto, Canadá retrocedió de la sexta posición de la muestra en 1998 a la décima en 2002, a la vez que México sufría un deterioro más pronunciado, cayendo del lugar 39 al 55. Si la degradación de la competitividad microeconómica de Canadá es atribuible a ambos subíndices, su punto débil se halla sobre todo en las prácticas y estrategias de las empresas; en el caso de México, es el deterioro del subíndice de calidad del contexto nacional de los negocios el que ha sido más pronunciado (México cae del puesto 41 en 1998, al 60 en 2002).

En síntesis, estas dos estimaciones de la competitividad internacional de Canadá y de sus socios del TLCAN muestran resultados parcialmente congruentes entre sí. Aun cuando las diferencias en las muestras de países y las metodologías aplicadas dificultan la comparación, podemos apreciar puntos comunes y divergencias en su apreciación de la competitividad de Canadá. En primer lugar, ambas coinciden en cuanto a la clasificación de la competitividad de los tres socios: Canadá se halla en una posición intermedia entre

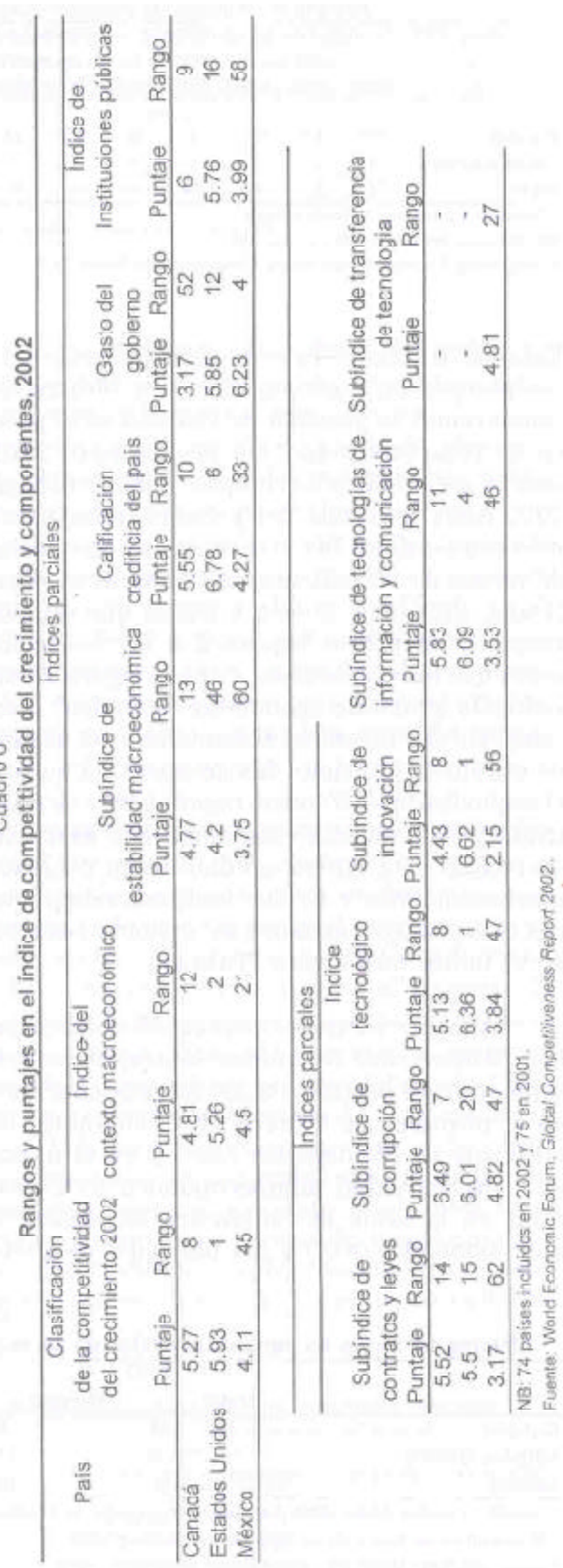


Cuadro 7

Rangos en el índice de competitividad microeconómica y componentes, 1998-2002

\begin{tabular}{|c|c|c|c|c|c|c|c|c|c|c|c|c|c|c|c|c|}
\hline \multirow[t]{2}{*}{ País } & \multicolumn{5}{|c|}{$\begin{array}{l}\text { Índice de competitividad } \\
\text { microeconómica }\end{array}$} & \multicolumn{5}{|c|}{$\begin{array}{c}\text { Clasificación de la estrategia y } \\
\text { operaciones empresariales }\end{array}$} & \multicolumn{5}{|c|}{$\begin{array}{l}\text { Clasificación de la calidad del } \\
\text { contexto nacional de negocios }\end{array}$} & \multirow{2}{*}{$\begin{array}{c}\text { PIB per cápita* } \\
2001 \\
\end{array}$} \\
\hline & 2002 & 2001 & 2000 & 1999 & 1998 & 2002 & 2001 & 2000 & 1999 & 1998 & 2002 & 2001 & 2000 & 1999 & 1998 & \\
\hline & 10 & 12 & 11 & 8 & 6 & 13 & 14 & 16 & 12 & 15 & 7 & 11 & 8 & 4 & 3 & \\
\hline Estados Unidos & 1 & 2 & 2 & 1 & 1 & 1 & 1 & 2 & 1 & 2 & 1 & 2 & 2 & 1 & 1 & 28611 \\
\hline México & 55 & 52 & 42 & 34 & 39 & 45 & 46 & 42 & 30 & 29 & 60 & 52 & 43 & 35 & 41 & 8969 \\
\hline
\end{tabular}

* Calculado a paridad de poder de compra.

NB: 74 países incluidos en 2002 y 75 en 2001

Fuente: World Economic Forum, Global Competitiveness Report 2002.

Estados Unidos - la economía juzgada más competitiva en ambos índices-y México. Si comparamos la posición de Canadá en el índice de competitividad del crecimiento 2002 (WEF) y en el índice de competitividad mundial 2002 (IMD), quitando del primero, para hacer más comparables las muestras, las economías de menos de 20 millones de habitantes -Finlandia, Singapur, Suecia y Suiza, que ocupan respectivamente los lugares $2,4,5$ y 6 - se observa que en ambos casos Canadá figura en un reducido grupo de economías "grandes" muy competitivas: en efecto, Canadá tiene el segundo puesto en el primer índice mientras que en el segundo ocupa el cuarto lugar, detrás de Australia y Taiwan, dos economías que destacan en cuanto a la buena calidad de su contexto macroeconómico y de sus instituciones públicas (Australia), y debido a su segunda posición en el índice tecnológico (Taiwan).

Algunos puntos comunes adicionales en las estimaciones de ambos informes pueden señalar áreas problemáticas: éstas se observan en el retroceso de Canadá en el subíndice de eficiencia de los negocios (wCY) y en el índice de competitividad microeconómica (GCR), así como en la caída del subíndice de eficiencia gubernamental (wCY) y del indicador de gasto gubernamental (wcy). Por otro lado, la mejora en el subíndice de infraestructura (wCY) contrasta en parte con la caída del subíndice tecnológico (GCR). Finalmente, cabe señalar que la construcción misma de estos índices y en especial las ponderaciones de las variables utilizadas determinan en parte el grado de estabilidad/volatilidad de los resultados: el índice del wcy presenta puntajes y rangos mucho más estables a través del tiempo, mientras que las variaciones año tras año de los dos índices del GCR son mucho más acentuadas debido al gran peso concedido a variables como la tecnología o las microeconómicas.

\section{La competitividad de Canadá frente a la IED}

El cálculo del índice de transnacionalidad de la UNCTAD, disponible hasta 1999, muestra que Canadá es la economía de América del Norte en la cual la IED desempeña el papel más destacado. En 1999, Canadá figuraba en séptimo lugar en este índice, detrás de economías desarrolladas más pequeñas (Bélgica-Luxemburgo, Irlanda, Suecia, Nueva Zelanda, los Países Bajos, Dinamarca). El papel de la IED se ha elevado en las tres economías del TLCAN entre 1997

Cuadro 8

Valores del índice de transnacionalidad de las economías receptoras elaborado por la UNCTAD, 1997-1999

\begin{tabular}{|c|c|c|c|c|c|c|}
\hline & \multicolumn{2}{|c|}{1997} & \multicolumn{2}{|c|}{1998} & \multicolumn{2}{|c|}{1999} \\
\hline & Valor & Rango* & Valor & Rango* & Valor & Rango* \\
\hline Canadá & 14 & 8 & 15.3 & 9 & 17.6 & 7 \\
\hline Estados Unidos & 6 & 21 & 7 & 21 & 8.2 & 20 \\
\hline México & 11 & 16 & 11 & 18 & 11.6 & 22 \\
\hline
\end{tabular}

* Canadá y Estados Unidos están clasificados en un grupo de 22 economías desarrolladas y México en un grupo de

30 economías en desarrollo en 1997-1998, y de 33 en 1999.

Fuente: UNCTAD, World Investment Report 2002, 2001, 2000. 
La competitividad externa de Canadá y de sus socios del TLCAN revelada en...

Cuadro 9

Componentes del índice de transnacionalidad de las economías receptoras (UNCTAD) en 1999

\begin{tabular}{lcccccc}
\hline & $\begin{array}{c}\text { Flujos de IED } \\
\text { (\% de la FBCF, } \\
\text { prom. 1997-99) }\end{array}$ & $\begin{array}{c}\text { Stock de IED } \\
\text { en el país } \\
\text { (\% del PIB) }\end{array}$ & $\begin{array}{c}\text { Afiliadas extranjeras } \\
\text { Valor agregado } \\
\text { /PIB }\end{array}$ & $\begin{array}{c}\text { Empleo/empleo } \\
\text { total }(\%)\end{array}$ & $\begin{array}{c}\text { Índice de } \\
\text { transnacionalidad }\end{array}$ & $\begin{array}{c}\text { Rango en el } \\
\text { índice }\end{array}$ \\
\hline Canadá & 16.1 & 26.5 & 15 & 12.8 & 17.6 & 7 \\
Estados Unidos & 12.8 & 10.5 & 4.9 & 4.5 & 8.2 & 20 \\
México & 14.2 & 16.3 & 8.8 & 7 & 11.6 & 22 \\
\hline
\end{tabular}

* Canadá y Estados Unidos están clasificados en un grupo de 22 economías desarrolladas y México en un grupo de

30 economías en desarrollo en 1997-1998, y de 33 en 1999.

Fuente: UNCTAD, World Investment Report 2002.

y 1999, pero su papel en México y Estados Unidos es relativamente menor (cuadro 8). En 1999 los flujos de IED representaban el $16.1 \%$ de la formación bruta de capital fijo (FBCF) en Canadá, contra $12.8 \%$ en Estados Unidos y $14.2 \%$ en México, y el stock de IED existente alcanzaba el $26.5 \%$ del PIB , una proporción a todas luces mayor que en Estados Unidos (10.5\%) y en México (16.3\%, ver cuadro 9).

La comparación de los dos siguientes índices arroja una luz diferente sobre la atracción realizada y potencial de IED de las tres economías. En primer lugar, el índice de desempeño de la IED muestra que en el período 1988-1990, las tres atrajeron una proporción de la IED global superior a la participación de sus PIB nacionales en el PIB global, con valores del índice superiores a 1. En 1998-2000, la situación cambia sustancialmente, al atraer tanto México como Estados Unidos una proporción de IED menor, con valores respectivos de 0.7 y 0.8, mientras Canadá eleva su participación relativa. En consecuencia, Canadá asciende al rango 30 de la clasificación, mientras Estados Unidos y México retroceden del puesto 50 al 74 y del 42 al 78, respectivamente. En ambos casos, estos resultados se pueden atribuir en parte a una aceleración del crecimiento económico que no fue seguida por un incremento proporcional de la IED.
Los resultados del cálculo del índice de potencial de IED son bastante diferentes: Estados Unidos y Canadá son la primera y la quinta economía con mayor potencial de atracción en 1998-2000 y, entre los dos períodos considerados, Canadá se ha deslizado desde el segundo lugar, lo cual indica una pérdida relativa de potencial, en tanto que México mejoraba su posición, del rango 77 al 70 . ¿A qué se pueden atribuir estos cambios de posicionamiento relativo? La cuadro 11, que muestra el desglose del índice para los dos períodos, permite plantear algunas hipótesis. En primer lugar, cabe recalcar que las tres economías, y en particular México, han ganado en cuanto a sus perspectivas de crecimiento (estimadas por el crecimiento real promedio del PIB), pero Estados Unidos ha afirmado en forma neta su ventaja en lo que concierne al tamaño del mercado interno, un criterio donde Canadá retrocede y México progresa poco. El criterio de apertura de la economía muestra que Canadá es la economía que más progresó al respecto, con exportaciones que representaban el $43.75 \%$ del PIB en 1998-2000 (contra 31.1\% para México y $11 \%$ para Estados Unidos), reforzando relativamente su capacidad de atraer IED sobre estas bases. Otro punto interesante es el que se refiere a los gastos de investigación y desarrollo, donde las diferencias entre Canadá y Estados Unidos se reducen, en razón de

Cuadro 10

Valores y rangos en los índices de desempeño y de potencial de la IED elaborados por la UNCTAD, 1988-1990 y $1998-2000$

\begin{tabular}{|c|c|c|c|c|c|c|c|c|}
\hline & \multicolumn{4}{|c|}{ Índice de desempeño de la IED } & \multicolumn{4}{|c|}{ Índice de potencial de la IED } \\
\hline & \multicolumn{2}{|c|}{ Valor } & \multicolumn{2}{|c|}{ Rango } & \multicolumn{2}{|c|}{ Valor $0-1$} & \multicolumn{2}{|c|}{ Rango } \\
\hline & $1988-1990$ & $1998-2000$ & $1988-1990$ & $1998-2000$ & $1988-1990$ & $1998-2000$ & $1988-1990$ & $1998-2000$ \\
\hline Canadá & 1.3 & 1.6 & 46 & 30 & 0.618 & 0.629 & 2 & 5 \\
\hline Estados Unidos & 1.1 & 0.8 & 50 & 74 & 0.649 & 0.666 & 1 & 1 \\
\hline México & 1.5 & 0.7 & 42 & 78 & 0.196 & 0.278 & 77 & 70 \\
\hline
\end{tabular}

Fuente: UNCTAD, World Investment Report 2002. 


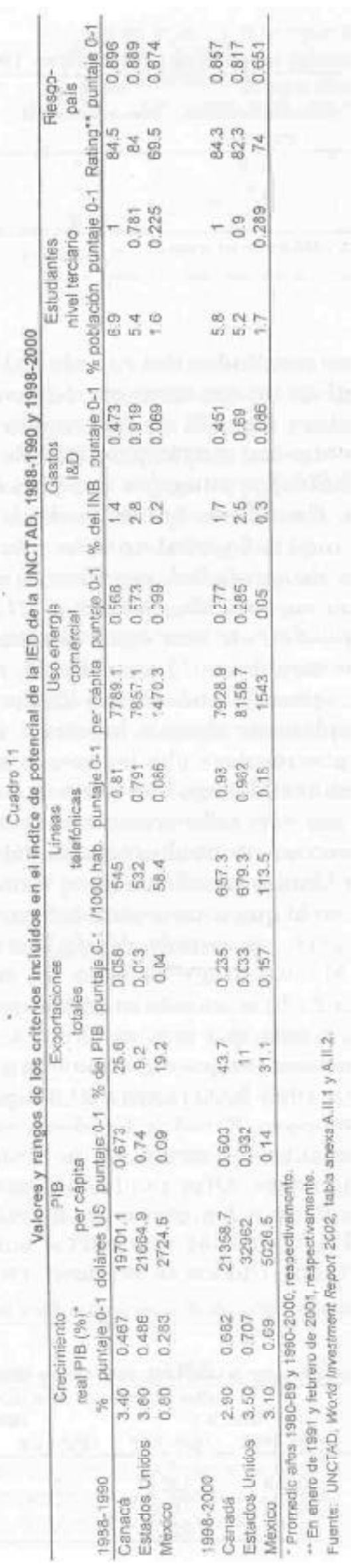

una elevación del gasto del primero combinada con un menor gasto del segundo, mientras México no logra reducir su desventaja competitiva en esta área. Finalmente, Canadá conserva la primacía entre las tres economías en cuanto al nivel de formación de su población, incluso a pesar de que la evolución de los valores del índice de desarrollo humano del PNUD muestra que ha ido perdiendo su ventaja frente a Estados Unidos a lo largo de los años (cuadro 12).

\section{Conclusión}

En este texto se analizó el tema de la competitividad externa de Canadá y de sus socios del TLCAN a partir de índices y demás indicadores internacionales creados para estimarla de manera empírica. Aun cuando no se intentó realizar una evaluación crítica de estos índices, pues el texto se limitó a una presentación muy general, hemos visto que tanto las metodologías aplicadas como las muestras de las economías abordadas difieren de manera considerable y que los resultados parecen ser bastante sensibles a estas diferencias iniciales. Ahora bien, no obstante el carácter parcial y ciertamente perfectible de las estimaciones de competitividad externa que ofrecen estos instrumentos empíricos, la amplia difusión de sus resultados y la gran atención que éstos reciben año tras año por parte de los medios, aunados a su utilización por las empresas como un apoyo para decidir, por ejemplo, las ubicaciones futuras de plantas productivas, les han otorgado cierto poder y capacidad de influencia.

Si bien las metodologías divergentes vuelven imposible toda comparación precisa de los resultados obtenidos, se pueden sin embargo sacar algunas conclusiones muy generales. La economía canadiense es de las más competitivas en el ámbito mundial, como lo muestran los datos proporcionados tanto por el WEF como por la escuela de negocios IMD en sus respectivas publicaciones. De acuerdo con los resultados presentados, las fortalezas de la economía canadiense residirían en sus instituciones públicas, su infraestructura física y humana, su estabilidad macroeconómica y la sustentabilidad ambiental de su modelo de 


\section{Cuadro 12}

Valores y rango del índice de desarrollo humano (IDH) del PNUD, 1975-2001

\begin{tabular}{lccccccc}
\hline & Ranqo 2001 & 1975 & 1980 & 1985 & 1990 & 1995 & 2001 \\
\hline Canadá & 8 & 0.866 & 0.881 & 0.904 & 0.924 & 0.929 & 0.937 \\
Estados Unidos & 7 & 0.864 & 0.883 & 0.896 & 0.911 & 0.923 & 0.937 \\
México & 55 & 0.684 & 0.729 & 0.748 & 0.757 & 0.771 & 0.8 \\
\hline
\end{tabular}

Fuente: PNUD, Human Development Report 2003.

desarrollo. En contraste, su desempeño macroeconómico reciente ha sido menos satisfactorio, y presenta desafíos en el área tecnológica y al nivel microeconómico, tanto en lo que concierne a las empresas mismas como al ámbito en el cual éstas se desempeñan.

Finalmente, los indicadores de la UNCTAD subrayan el carácter transnacionalizado de la economía canadiense, así como su alto grado de apertura comercial. En ambos casos, estos indicadores se han elevado notablemente entre $1988-1990$ y $1998-2000$, mostrando el fuerte impacto del acuerdo comercial con Estados Unidos, seguido a partir de 1994 por el TLCAN.

\section{Notas}

1 El anuario se publica en forma continua desde 1989 y el índice desde 1995.

2 Por esta razón también, el desempeño económico de las economías en desarrollo se entiende a largo plazo, es decir, una vez descontado el componente relacionado con el catching-up o proceso de recuperación del retraso.

3 Véase Stéphane Garelli, "Competitiveness of nations: the fundamentals", en World Competitiveness Yearbook 2003 y Suzanne Rosselet-McCaulay, "Methodology and principles of analysis", en http://www01.imd.ch/wcy, para una presentación detallada de los supuestos que presiden a la construcción del índice y la metodología empleada.

4 Estas economías se definen como aquellas que depositaron más de quince patentes estadounidenses de utilidad por millón de habitantes en 2001. A la lista inicial, que comprendía 18 economías de América del Norte y de Europa, siendo Israel, Japón, Australia y Nueva Zelanda los únicos representantes de otros continentes, se han agregado en el año 2000 dos economías europeas (Islandia e Irlanda) y los cuatro "tigres" asiáticos: Hong Kong, Corea del Sur, Singapur y Taiwan, véase Peter K. Cornelius, Jennifer Blanke y Fiona Paua, "The Growth Competitiveness Index: recent economic developments and the prospects for a sustained recovery", en Global Competitiveness Report 2003 (página 10 , tabla 2 ).

5 La composición del índice tecnológico varía también entre economías innovadoras centrales, donde se da especial atención a la innovación (mitad de la ponderación del índice), y las demás, donde entran las transfe- rencias de tecnología (3/8 de la ponderación) antes de la innovación propia (1/8).

6 El informe recalca que en cada nivel de desarrollo, las exigencias y desafíos se irán modificando, produciéndose "puntos de inflexión" en la transición de un nivel a otro, en los cuales numerosos aspectos estrechamente relacionados de la competencia tendrán que ser transformados de manera fundamental.

7 Véase en Heritage Foundation, 2003 Index of Economic Freedom, en <http://www.heritage.org/research/features/ index>, (2003: cap. 5) los detalles de la elaboración del índice y, en particular, de los criterios utilizados para atribuir el puntaje a cada aspecto revisado.

$8 \mathrm{El}$ informe proporciona información de la IED para un número mayor de países, pero sólo calcula los índices para un grupo más reducido.

9 Véanse wef, Canada: Competitiveness Profile, United States: Competitiveness Profile, Mexico: Competitiveness Profile, para el año 2001, en http://www.weforum.org/ site/knowledgenavigator.nsf/Content.

\section{Referencias bibliográficas}

Cornelius, Peter K., Jennifer Blanke y Fiona Paua: "The Growth Competitiveness Index: recent economic developments and the prospects for a sustained recovery", en weF, Global Competitiveness Report 2002.

Garelli, Stéphane "Competitiveness of nations: the fundamentals", en IMD, World Competitiveness Yearbook 2003.

Heritage Foundation: 2003 Index of Economic Freedom, en <http://www.heritage.org/research/features/index> IMD World Competitiveness Yearbook 2003.

Porter, Michael E. "Building the microeconomic foundations of prosperity: findings from the Microeconomic Competitiveness Index", en weF, Global Competitiveness Report 2002.

Rosselet-McCaulay, Suzanne "Methodology and principles of analysis", en http://www01.imd.ch/wcy, consultado en agosto 2003.

UNCTAD. Word Investment Report 2002.

UNCTAD. Word Investment Report 2000.

wEF. Global Competitiveness Report 2002. T:" 